\title{
ENSINO REMOTO DE URGÊNCIA NOS CURSOS DA ÁREA DA SAÚDE DURANTE O DISTANCIAMENTO SOCIAL GERADO PELA PANDEMIA
}

\author{
ANA CAROLINA DONDONI FÁVERO ${ }^{1}$, FELIPE MOURA PARREIRA ${ }^{2}$
}

\begin{abstract}
1 Graduanda de Medicina, Centro Universitário UNIFACIG, carool_favero@ hotmail.com
2 Mestrando em Desenvolvimento Local, Pós-graduado em Docência em Ciências da Saúde e Perícias Médicas, MBA em Gestão de Equipes e Liderança, Especialista em Clínica Médica e Reumatologia, Graduado em Medicina pela UNEC, felipemouraparreira@ hotmail.com
\end{abstract}

\section{RESUMO}

Com o avanço do novo Coronavírus, as restrições exigidas pelo distanciamento social geraram impactos sobre as atividades da população, principalmente sobre as escolas e universidades, que têm experimentado um novo modelo educacional, marcado pela mudança da educação presencial para o ensino on-line. $\mathrm{O}$ ensino remoto emergencial traz consigo uma preocupação em relação à carência de fiscalização, podendo comprometer a qualidade do ensino, prejudicando a formação dos futuros profissionais. Este trabalho trata de uma pesquisa de levantamento, utilizando como instrumento de coleta de dados o survey, aplicado em meio digital. A população escolhida foram acadêmicos dos cursos da área da saúde de um Centro Universitário do leste mineiro sujeitos ao ensino totalmente on-line durante o período de pandemia. Após o recebimento das respostas, os dados foram analisados, sendo que, dos resultados obtidos, $72,7 \%$ dos estudantes acreditam que o ensino remoto pode ser uma ferramenta complementar ao ensino presencial, enquanto $27,3 \%$ acredita que não. Quando questionados se professores estão capacitados para o emprego do ensino remoto, $59 \%$ afirmaram que sim, mas $41 \%$ acredita que não. Em relação à produtividade, $87,5 \%$ dos estudantes afirmaram que as aulas presenciais são mais produtivas que as aulas à distância, enquanto $12,5 \%$ acredita que o ensino remoto seja mais produtivo. Os maiores dificultadores técnicos das aulas on-line foram: internet inadequada $(52,4 \%)$, local inadequado para assistir às aulas $(22,9 \%)$ e recursos tecnológicos inadequados $(8,5 \%)$. Ao serem interrogados sobre seu nível de satisfação com o ensino remoto, as respostas dos acadêmicos variaram desde totalmente satisfeitos a totalmente insatisfeitos. Em virtude dos aspectos observados, depreende-se que o ensino remoto implantado no contexto da pandemia é uma solução imediata que pode continuar sendo empregada junto ao ensino presencial no intuito de enriquecer o crescimento científico e possibilitar maiores acessos.

Palavras-Chave: Ensino Remoto; Ensino Superior; Docência; Pandemia.

\section{REMOTE EMERGENCY TEACHING IN HEALTHCARE COURSES DURING THE SOCIAL DISTANCING GENERATED BY PANDEMIA}




\begin{abstract}
With the advancement of the new Coronavirus, the restrictions demanded by social distance have generated impacts on the activities of the population, mainly on schools and universities, which have been experiencing a new educational model, marked by the change from classroom education to online teaching. Emergency remote education brings with it a concern regarding the lack of inspection, which may compromise the quality of teaching, jeopardizing the training of future professionals. This work is a survey with digital survey. The population chosen were academics from health courses at a University Center in eastern Minas Gerais, subject to fully online education during the pandemic period. Data collection was performed using a virtual questionnaire sent to academics. After receiving the answers, the data were analyzed. $72.7 \%$ of students believe that remote education can be a complementary tool to classroom teaching, while $27.3 \%$ believe that it cannot. When asked if teachers are qualified to use remote education, $59 \%$ said yes, but $41 \%$ believed not. Regarding productivity, $87.5 \%$ of the students stated that the face-to-face classes are more productive than distance classes, while $12.5 \%$ believe that remote teaching is more productive. The biggest technical difficulties in online classes were: inadequate internet $(52.4 \%)$, inadequate place to attend classes $(22.9 \%)$ and inadequate technological resources $(8.5 \%)$. When asked about their level of satisfaction with remote education, the students' responses ranged from totally satisfied to totally dissatisfied. In view of the aspects observed, it appears that remote education implemented in the context of the pandemic is an immediate solution that can continue to be used with face-to-face education in order to enrich scientific growth and enable greater access.
\end{abstract}

Keywords: Remote Teaching; University education; Teaching; Pandemic.

\title{
1 INTRODUÇÃO
}

Desde março de 2020, devido às restrições exigidas pelo distanciamento social decorrente da disseminação do novo Coronavírus (SARS COV2), escolas e universidades do mundo todo têm experimentado um novo modelo educacional, marcado pela mudança da educação tradicional presencial em sala de aula para o ensino on-line (EL KHATIB, 2020).

Além do Brasil, 187 países foram atingidos pela pandemia e, em um pequeno intervalo de tempo, milhares de educadores precisaram criar alternativas para ensinar à distância (JOHNS HOPKINS UNIVERSITY, 2020). Utilizada anteriormente em menor escala, a videoconferência é hoje a principal ferramenta de aprendizagem e tem sido muito usada a fim de facilitar a comunicação entre discentes e docentes ou alunos e seus colegas (TORRES; ALVES; COSTA, 2020). 
Porém, a efetividade do ensino remoto depende da interação de vários fatores, desde o perfil do estudante e a sua determinação até a preparação e formação do corpo docente em recursos tecnológicos de apoio a essa modalidade de estudo (SILVA, 2020).

De acordo com Lawson et al. (2010), o ensino remoto, quando enfatizado em conteúdos específicos, pode ser uma maneira de estudo eficaz, principalmente para alunos autodidatas e mais comprometidos. Em contrapartida, se essa forma de ensino se mantiver por um período relativamente prolongado, os alunos podem piorar seus resultados, diminuindo, dessa forma, a possibilidade de sucesso escolar (LAWSON et al., 2010).

É importante lembrar que o ensino remoto implantado durante a pandemia assemelha-se ao ensino à distância apenas por serem modalidades de educação mediadas pela tecnologia; os princípios do ensino remoto continuam sendo os mesmos do ensino presencial (JOYE; MOREIRA; ROCHA, 2020).

O ensino remoto emergencial adotado pelas escolas e universidades traz consigo uma preocupação em relação à escassez de fiscalização que ele apresenta, podendo comprometer a qualidade do ensino, prejudicando a médio e longo prazo a formação dos futuros profissionais (TORRES; ALVES; COSTA, 2020).

O presente artigo sobre o tema "Ensino remoto de urgência durante o distanciamento social gerado pela pandemia" tem por objetivo refletir sobre a eficácia da educação em cursos superiores da área da saúde durante a pandemia causada pelo COVID-19 e justifica-se pela necessidade de discutir sobre os modelos de ensino sugeridos de forma emergencial no contexto da pandemia.

\section{METODOLOGIA}

Trata-se de um estudo do tipo descritivo com abordagem qualitativa, do tipo pesquisa de levantamento com survey por meio digital visando esclarecer a pergunta norteadora: o ensino remoto de urgência é uma solução para o distanciamento social provocado pela pandemia?

A pesquisa com survey é a pesquisa que busca informações sobre as características ou as opiniões de determinado grupo de interesse a respeito dos dados 
que se deseja obter utilizando um questionário como instrumento de pesquisa (GIL, 2007).

A população escolhida para a realização da pesquisa foi constituída por acadêmicos dos cursos da área da saúde de um Centro Universitário do leste mineiro sujeitos ao ensino totalmente on-line durante o período de isolamento social. Essa instituição de ensino conta com 245 acadêmicos do curso de Medicina, 145 de Enfermagem, 120 de Psicologia e 265 de Odontologia, somando 775 alunos matriculados na área da saúde.

A coleta de dados foi realizada utilizando-se um questionário virtual por meio da ferramenta on-line Google Forms.

O questionário foi estruturado com base em algumas variáveis como o nível de satisfação dos alunos com as aulas remotas, as dificuldades técnicas encontradas, a produtividade em relação às aulas presenciais, a capacitação dos professores para o uso do ensino remoto e a possibilidade de ser uma ferramenta complementar ao ensino presencial.

O formulário foi enviado para os acadêmicos no dia 31 de julho de 2020, sendo estipulado um período de duas semanas para o recebimento das respostas. Encerrado esse período, os dados coletados foram analisados. Responderam ao questionário 271 membros do corpo discente, correspondendo a aproximadamente $35 \%$ do total dos estudantes da área da saúde.

A pesquisa levou em conta a preservação dos princípios éticos e legais que regem a pesquisa em seres humanos, recomendados na Resolução n. 510/2016 do Conselho Nacional de Saúde (CNS) do Ministério da Saúde, que determina serem dispensáveis submissões ao Comitê de Ética em Pesquisas que utilizem informações de opinião pública.

\section{RESULTADOS E DISCUSSÃO}

O propósito deste estudo foi analisar o andamento do ensino on-line durante a pandemia através da identificação dos fatores positivos e negativos apontados pelos acadêmicos. Os resultados estão sendo apresentados a partir dos dados obtidos no questionário. 
Buscou-se, inicialmente, investigar se os acadêmicos enxergam o ensino remoto como um instrumento complementar ao ensino presencial. Os dados estão representados no Gráfico 1.

GRÁFICO 1 - O ensino remoto pode ser uma ferramenta complementar ao ensino presencial?

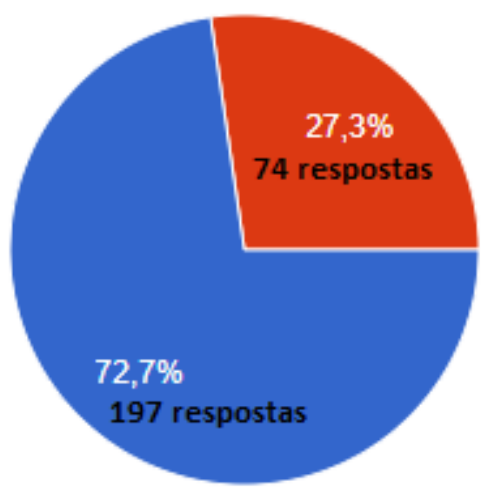

Fonte: Dados da pesquisa

Conforme se observa no Gráfico 1, 197 estudantes (72,7\%) acreditam que o ensino remoto pode ser uma ferramenta complementar ao ensino presencial, enquanto somente $74(27,3 \%)$ discordam e acreditam que não são complementares.

Nesse seguimento, é importante aproveitar o interesse natural dos jovens pelas tecnologias e inovações e empregá-las para transformar a sala de aula on-line em um ambiente de aprendizagem ativa. Mill (2013) acredita que essa geração de estudantes tem consciência de que é preciso estar adaptado ao ensino à distância, pois as novas exigências do mundo do trabalho englobam produção e manipulação de informações no ambiente virtual e, por isso, seria interessante implantar um ensino híbrido diante dessa nova realidade.

Além disso, o uso das tecnologias contemporâneas de comunicação e informação tem contribuído para a descentralização dos processos educacionais, viabilizando a comunicação entre acadêmicos de classes e lugares diferentes. Dessa maneira, a evolução do aprendizado transcende os espaços físicos de ensino e permeia as atividades sociais (BEZERRA et al., 2020). As novas tecnologias também podem 
auxiliar na minimização de algumas limitações das aulas presenciais e promover a inclusão digital do corpo discente (TAKAHASHI; CARDOSO, 2011).

Em seguida, buscou-se compreender qual é a percepção dos acadêmicos em relação à capacitação do corpo docente para as aulas online. O Gráfico 2 representa os dados obtidos.

GRÁFICO 2 - Os professores estão capacitados para o uso do ensino remoto?

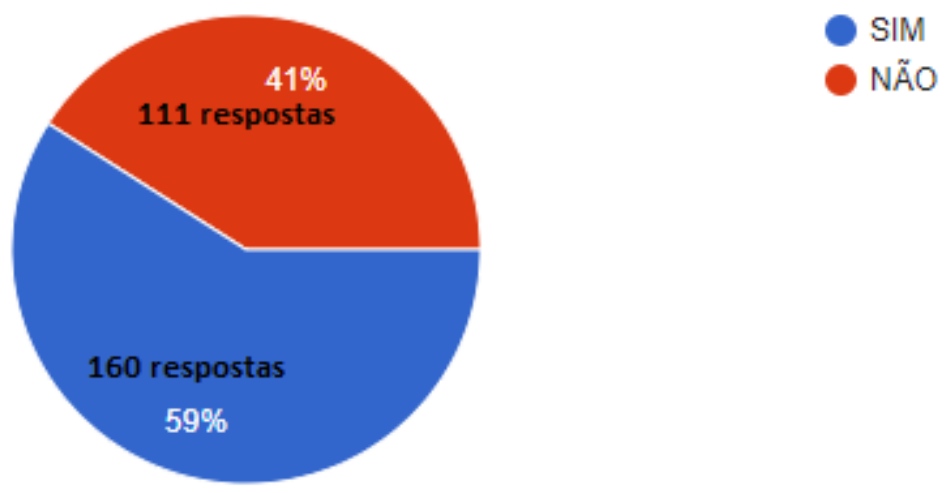

Fonte: Dados da pesquisa

De acordo com o Gráfico 2, quando questionados se os professores estão capacitados para o emprego do ensino remoto, $59 \%(n=160)$ dos acadêmicos afirmaram que sim, mas $41 \%(n=111)$ acredita que o corpo discente não está qualificado para ministrar aulas à distância.

Diante desses dados, percebe-se que é necessário maior engajamento dos educadores e da Instituição de Ensino frente ao desafio de ensinar à distância. É primordial que haja interesse mútuo em reestruturar ementas, explorar as ferramentas digitais, empregar metodologias ativas, motivar os alunos para a aprendizagem durante o período de aulas remotas e propor inovações pedagógicas aos professores. Dessa maneira, tanto os professores quanto a Instituição de Ensino estariam alinhados e preparados para oferecer aos estudantes um ensino de qualidade (ROSO et al., 2015).

As Instituições de Ensino precisam estar atentas a esta situação, pois não se trata apenas de simples substituições metodológicas, mas sim de importantes modificações 
no contexto de ensino que devem levar em consideração a complexidade dos encargos do professor e suas obrigações de trabalho (LOBO; MAIA, 2015).

Dando sequência ao questionário, os acadêmicos foram perguntados se as aulas presenciais são mais produtivas que as aulas on-line. $\mathrm{O}$ resultado está ilustrado no Gráfico 3.

GRÁFICO 3 - As aulas presenciais são mais produtivas que as aulas on-line?

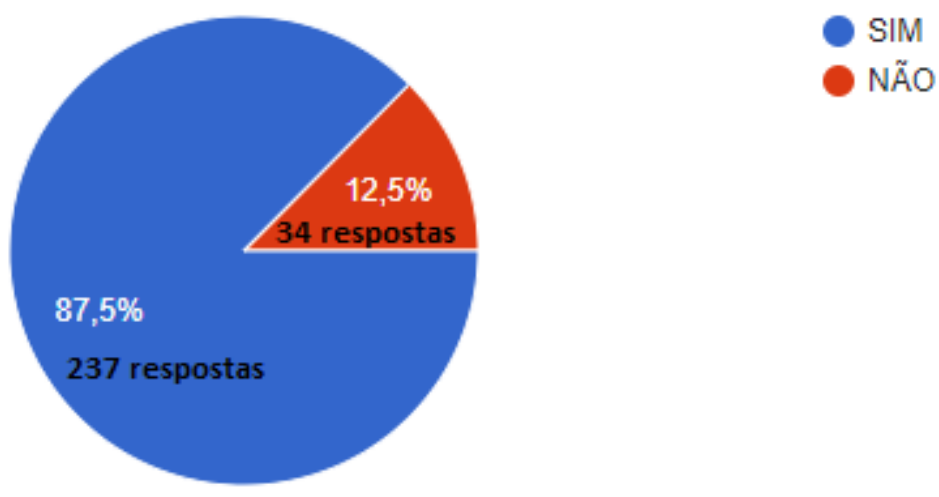

Fonte: Dados da pesquisa

Em relação à produtividade, 237 estudantes $(87,5 \%)$ afirmaram que as aulas presenciais são mais produtivas que as aulas on-line, enquanto a pequena minoria de $12,5 \%(n=34)$ acredita que o ensino remoto seja mais produtivo.

A queda da produtividade relatada pela ampla maioria dos estudantes durante o ensino remoto pode ser explicada, segundo Brooks et al. (2020), pelos maiores níveis de estresse, ansiedade, apatia e desânimo provocados pelo isolamento social, refletindo negativamente no rendimento acadêmico. Por conta disso, é extremamente importante que haja um reforço na detecção e solução das adversidades psicossociais através da implantação de mecanismos de adaptabilidade e de medidas para avaliar o bem-estar e a saúde mental dos acadêmicos (FERREIRA et al., 2020).

Além disso, os universitários trazem consigo um modelo de ensino médio em que o professor dita o conteúdo e os alunos estudam estritamente apenas aquele assunto. Na nova realidade de ensino, o professor adota diferentes posturas e ações, transferindo a responsabilidade da busca pelo conhecimento aos estudantes; este processo exige 
disciplina e dedicação e pode ser dificultado durante o período de aulas remotas, refletindo na queda do rendimento escolar (FERRARI; SEKKEL, 2007).

Diversos fatores influenciaram no processo de ensino; diante disso, buscou-se compreender o posicionamento dos estudantes quanto às dificuldades técnicas encontradas durante as aulas online, como mostra o Gráfico 4.

GRÁFICO 4 - Qual foi o maior dificultador técnico das aulas on-line?

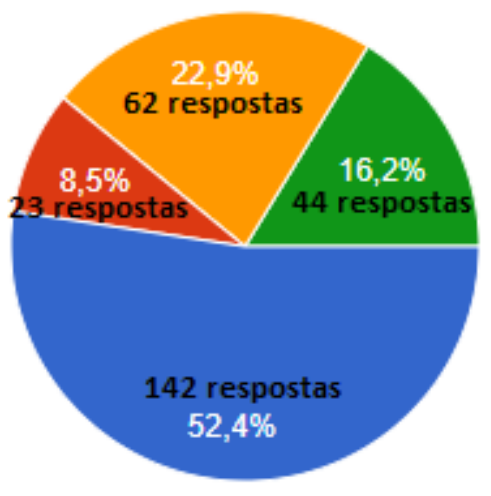

Internet inadequada

Computador inadequado (associa-se fones e etc)

Local inadequado para assistir às aulas

Não tive dificultadores técnicos

Fonte: Dados da pesquisa

De acordo com o Gráfico 4, a maior dificuldade técnica das aulas on-line durante a pandemia foi a internet inadequada $(52,4 \%)$, seguida de local inadequado para assistir às aulas $(22,9 \%)$ e recursos tecnológicos e de mídia - como computadores e acessórios - inadequados (8,5\%). Em contrapartida, 16,2\% dos acadêmicos não tiveram dificultadores técnicos durante as aulas online.

Evidentemente, alunos e educadores precisaram se adaptar às novas rotinas e à nova sala de aula, conciliando os espaços em casa. A implantação do ensino remoto envolveu muitas dificuldades, dentre elas a falha de tecnologia, a carência de orientação educativa específica e, ainda, uma certa resistência por parte dos acadêmicos sujeitos a aceitar esse novo formato de ensino (BUTTIGNON; GARCIA; SILVA, 2002).

Um estudo realizado por Borba et al. (2020) com o intuito de compreender a situação do ensino durante o isolamento social gerado pela pandemia apontou que $66,7 \%$ dos professores e 55,6\% dos alunos apresentavam conexão de internet lenta ou instável. Isso demonstra que problemas como a qualidade insatisfatória das conexões e 
as oscilações dos serviços de internet são obstáculos frequentemente encontrados pelo corpo docente e discente (BORBA et al., 2020).

O local inapropriado foi apontado, em segundo lugar, como dificultador das aulas à distância. Por estarem restritos a um mesmo ambiente, é recorrente que as dinâmicas da casa causem transtornos aos acadêmicos durante as aulas on-line; além disso, muitas vezes, o computador é compartilhado pela família, o que dificulta o processo de continuidade do estudo (BORBA et al., 2020).

Por fim, considerou-se relevante obter informações sobre o nível de satisfação dos estudantes com as aulas à distância. O Gráfico 5 mostra os resultados.

GRÁFICO 5 - Qual é o seu nível de satisfação com o ensino remoto?

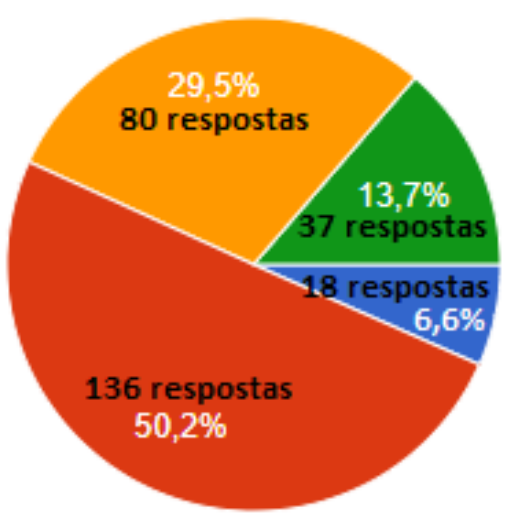

Totalmente Satisfeito

Parcialmente Satisfeito

Parcialmente Insatisfeito

Totalmente Insatisfeito

Fonte: Dados da pesquisa

Como ilustrado no Gráfico 5, os estudantes foram interrogados sobre seu nível de satisfação com o ensino remoto; 50,2\% (n=136) mostraram-se parcialmente satisfeitos, 29,5\% (n=80) parcialmente insatisfeitos, 13,7\% (37) totalmente insatisfeitos e $6,6 \%(n=18)$ totalmente satisfeitos.

De acordo com os dados, é possível perceber que mais de $50 \%$ dos estudantes expõe algum grau de satisfação com o ensino remoto. Segundo Moore e Kearsle (2007), isso acontece porque o distanciamento das atividades presenciais representa uma oportunidade ofertada ao estudante de aperfeiçoar o seu processo de aprendizagem de maneira autônoma. Ou seja, a autonomia é uma meta a ser alcançada pelos alunos, 
como uma evidência de maturidade, compromisso e disciplina para o aprendizado (MARQUES, 2020).

Além disso, as aulas gravadas possibilitam aos acadêmicos terem acesso aos conteúdos em outros momentos, viabilizando a flexibilização dos horários e, consequentemente, otimizando o aprendizado e a realização das atividades (FERREIRA et al., 2020).

Em contrapartida, considera-se que a insatisfação dos estudantes pode estar relacionada à dificuldade de manter uma rotina de estudo, seja por má adequação às aulas on-line, pelo receio em tirar dúvidas devido à baixa interação nesta modalidade de ensino ou até mesmo por saudades das aulas presenciais e dos amigos (MARQUES, 2020).

De qualquer forma, é importante direcionar e orientar os alunos para a adequação a esse formato de busca pelo conhecimento, pois é uma realidade que se faz cada vez mais presente nas atividades na vida contemporânea (KENSKI, 2005).

\section{CONCLUSÃO}

Ao final da pesquisa, constatou-se que a pergunta norteadora foi confirmada, pois, frente ao atual momento, o ensino remoto implantado no contexto da pandemia é uma solução imediata que apresentou mais resultados positivos do que negativos. Isso mostra que esta modalidade de ensino pode continuar sendo empregada junto ao ensino presencial no intuito de enriquecer o crescimento científico e possibilitar maiores acessos.

Como já relatado anteriormente, por mais que alguns alunos encarem o ensino remoto de urgência como uma maneira de exercer a autonomia, muitos discentes sentem-se vulneráveis inseridos nessa modalidade de ensino.

Por conta disso, é necessário que as Instituições de Ensino desenvolvam processos educativos que melhorem a qualidade profissional dos professores, definindo políticas e criando programas de capacitação, principalmente projetos de formação e educação digital que possibilitem realizar a adequada efetivação deste ensino. É importante que os encontros não se resumam somente a aulas on-line; deve-se 
diversificar as experiências de aprendizagem através do emprego de metodologias ativas e da diversificação dos canais de comunicação.

Além disso, o envolvimento das famílias é indispensável e deve ser estimulado nesse momento, desde que orientado por um olhar realista e minucioso.

\section{REFERÊNCIAS}

BEZERRA, K. P. et al. Ensino remoto em universidades públicas estaduais: o futuro que se faz presente. Research, Society and Development, v. 9, n. 9, p. e359997226e359997226, 2020. Disponível em: < https://rsdjournal.org/index.php/rsd/article/view/7226/6517>. Acesso em: 05 Nov. 2020.

BORBA, R. C. N. et al. Percepções docentes e práticas de ensino de ciências e biologia na pandemia: uma investigação da Regional 2 da SBEnBio. Revista de Ensino de Biologia da SBEnBio, v. 13, n. 1, p. 153-171, 2020. Disponível em: < http://sbenbio.journals.com.br/index.php/sbenbio/article/view/337/100>. Acesso em: 23 ago. 2020.

BUTTIGNON, K.; GARCIA, R. P. C.; SILVA, M. H. Uma Reflexão sobre o Ensino a Distância, via Internet, no Brasil. http://www.

feg.unesp.br/ceie/Monografias/CEIE0204.pdf, v. 25, n. 07, p. 07, 2002. Disponível em: $<$ http://www.conhecer.org.br/download/cp/NOVAS\%20TECNOLOGIAS/M3/leitura\%20 anexa\%203.pdf>. Acesso em: 23 ago. 2020.

BRASIL. Resolução no 510, de 07 de abril de 2016. Dispõe sobre as normas aplicáveis a pesquisas em Ciências Humanas e Sociais. Diário Oficial [da] República Federativa do Brasil, Brasília, DF, 24 maio 2016. Disponível em: <https://bvsms.saude.gov.br/bvs/saudelegis/cns/2016/res0510_07_04_2016.html>. Acesso em: 19 jul 2020.

BROOKS, S. K. et al. The psychological impact of quarantine and how to reduce it: rapid review of the evidence. The Lancet, 2020. Disponível em: <https://www.thelancet.com/action/showPdf?pii=S0140-6736\%2820\%2930460-8>. Acesso em: 17 ago. 2020.

COVID-19 Dashboard by the Center for Systems Science and Engineering (CSSE) at Johns Hopkins University (JHU). Disponível em: < https://gisanddata.maps.arcgis.com/apps/opsdashboard/index.html\#/bda7594740fd402994 23467b48e9ecf6>. Acesso em: 16.Jul.2020.

EL KHATIB, A. S. Aulas por Videoconferência: Uma solução para o distanciamento social provocado pela COVID-19 ou um grande problema? Disponível em: <https://preprints.scielo.org/index.php/scielo/preprint/view/787/1207>. Acesso em: 16.Jul.2020. 
FERRARI, M. A. L. D.; SEKKEL, M. C. Educação inclusiva no ensino superior: um novo desafio. Psicologia Ciência e Profissão, v. 27, n. 4, p. 636-647, 2007. Disponível em: < https://www.scielo.br/pdf/pcp/v27n4/v27n4a06>. Acesso em: 05 Nov. 2020.

FERREIRA, A. M. S. et al. COVimpact: pandemia COVID-19 nos estudantes do ensino superior da saúde. Revista de Investigação \& Inovação em Saúde, v. 3, n. 1, p. 7-16, 2020. Disponível em: <http://riis.essnortecvp.pt/index.php/RIIS/article/view/80/64>. Acesso em: 17 ago. 2020.

GIL, A. C. Como elaborar projetos de pesquisa. 4. ed. São Paulo: Atlas, 2007.

JOYE, C. R.; MOREIRA, M. M.; ROCHA, S. S. D. Educação a Distância ou Atividade Educacional Remota Emergencial: em busca do elo perdido da educação escolar em tempos de COVID-19. Research, Society and Development, v. 9, n. 7, p. e521974299e521974299, 2020. Disponível em: <

https://rsdjournal.org/index.php/rsd/article/view/4299/3757>. Acesso em: 29 Out. 2020.

KENSKI, V. M. Gestão e uso das mídias em projetos de educação a distância. Revista ecurriculum, v. 1, n. 1, 2005. Disponível em:

<https://revistas.pucsp.br/index.php/curriculum/article/view/3099/2042>. Acesso em: 03 Dez. 2020.

LAWSON, T. et al. Images of the future for education? Videoconferencing: A literature review. Technology, pedagogy and education, v. 19, n. 3, p. 295-314, 2010. Disponível em: <https://www.tandfonline.com/doi/abs/10.1080/1475939X.2010.513761>. Acesso em: 16.Jul.2020.

LOBO, A. S. M.; MAIA, L. C. G. O uso das TICs como ferramenta de ensinoaprendizagem no Ensino Superior. Caderno de Geografia, v. 25, n. 44, p. 16-26, 2015. Disponível em: < https://www.redalyc.org/pdf/3332/333239878002.pdf>. Acesso em: 05 Nov. 2020.

MARQUES, R. A ressignificação da educação e o processo de ensino e aprendizagem no contexto de pandemia da COVID-19. Boletim de Conjuntura (BOCA), v. 3, n. 7, p. 3146, 2020. Disponível em: < https://revista.ufrr.br/boca/article/view/Marques>. Acesso em: 16 ago. 2020.

MILL, D. Análise da educação a distância como interseção entre a formação docente, as tecnologias digitais e a pós-graduação. Educação em Perspectiva, v. 4, n. 2, p. 1-27, 2013. Disponível em:

<https://periodicos.ufv.br/educacaoemperspectiva/article/view/6621/2727>. Acesso em: 16 ago. 2020.

MOORE, M. G. KEARSLE, G. Educação a distância: uma visão integrada. São Paulo: Thomson, 2007.

ROSO, C. C. et al. Currículo temático fundamentado em Freire-CTS: engajamento de professores de física em formação inicial. Ensaio Pesquisa em Educação em Ciências, 
Belo Horizonte, v. 17, n. 2, p. 372-389, 2015. Disponível em: <https://www.scielo.br/pdf/epec/v17n2/1983-2117-epec-17-02-00372.pdf>. Acesso em: 16 ago. 2020.

SILVA, O. D. L. A Estatística e o Ensino Superior em regime não presencial no período da pandemia por Covid-19. Correio dos Açores, p. 17-17, 2020. Disponível em: < https://repositorio.uac.pt/bitstream/10400.3/5526/1/Silva_30_04_2020.pdf >. Acesso em: 16.Jul.2020.

TORRES, A. C. M.; ALVES, L. R. G.; COSTA, A. C. N. Educação e Saúde: reflexões sobre o contexto universitário em tempos de COVID-19. 2020. Disponível em: < https://preprints.scielo.org/index.php/scielo/preprint/view/640/885>. Acesso em: 16.Jul.2020.

TAKAHASHI, E. K.; CARDOSO, D. C. Experimentação remota em atividades de ensino formal: um estudo a partir de periódicos Qualis A. Revista Brasileira de Pesquisa em Educação em Ciências, v. 11, n. 3, p. 185-208, 2011. Disponível em: < https://periodicos.ufmg.br/index.php/rbpec/article/view/4214/2779>. Acesso em: 05 Nov. 2020. 